\title{
Cuidados paliativos em pediatria: uma revisão
}

Maria Thereza Macedo Valadares ${ }^{1}$, Joaquim Antônio César Mota ${ }^{2}$, Benigna Maria de Oliveira ${ }^{3}$

\section{Resumo}

A expansão tecnológica das últimas décadas modificou o perfil dos pacientes na pediatria, tornando cada vez mais frequente a assistência a crianças com doenças crônicas e ameaçadoras à vida. Cuidados paliativos envolvem a assistência ativa e total prestada a essas crianças, nas dimensões de seu corpo, mente e espírito, bem como o suporte oferecido a sua família. O presente estudo revisa aspectos relevantes na abordagem paliativa: a prevalência e abordagem dos sintomas, a participação de equipe multiprofissional, a comunicação com a família, os cuidados ao final da vida e a limitação de terapêuticas invasivas. A despeito do reconhecimento da importância da medicina paliativa, nossas universidades ainda priorizam a medicina curativa. Instituir esse tema no processo de formação do médico é uma necessidade real e absoluta, visando proporcionar vida e morte dignas aos nossos pacientes.

Palavras-chave: Cuidados paliativos. Dor. Assistência. Criança.

\section{Resumen}

\section{Los cuidados paliativos en pediatría: una revisión}

La expansión tecnológica de las últimas décadas ha cambiado el perfil de los pacientes de pediatría, volviendo cada vez más frecuente la asistencia a los niños con enfermedades crónicas y potencialmente mortales. Cuidados paliativos involucran la asistencia activa y total prestada a estos niños y niñas, en el ámbito de su cuerpo, mente y espíritu, así como el apoyo ofrecido a toda su familia. Este estudio proporciona un análisis de los aspectos relevantes en el enfoque paliativo: la prevalencia y abordaje de los síntomas, la participación de un equipo multidisciplinario, la comunicación con la familia, la atención al final de la vida y la limitación de las terapias invasivas. A pesar del reconocimiento de la importancia de la medicina paliativa, nuestras universidades siguen dando prioridad a la medicina curativa. Establecer ese tema en el proceso de la educación médica es una necesidad real y absoluta, con el objetivo de proporcionar una vida y una muerte dignas a nuestros pacientes.

Palabras-clave: Cuidados paliativos. Dolor. Atención. Niños.

\begin{abstract}
Palliative care in pediatrics: a review

The technological expansion in recent decades changed the profile of the patients in pediatrics, with assistance to children with chronic and life-threatening conditions more frequent each time. Palliative care involves an active and full assistance provided to these children, in the context of their body, mind and spirit as well as the support offered to their entire family. This study provides a review of relevant aspects in palliative approach: the prevalence and approach of symptoms, the participation of a multidisciplinary team, communication with family, care at end of life and limitation of invasive therapies. Despite recognition of the importance of palliative medicine, our universities still give priority to curative medicine. Introducing this theme in the process of medical education is a real and absolute need, aiming to provide dignified life and death to our patients. Key words: Palliative care. Pain. Assistance. Child.
\end{abstract}

1. Mestre therezaval@yahoo.com.br 2. Doutor jacmota@uol.com.br 3. Doutora benigna@uol.com.br - Universidade Federal de Minas Gerais, Belo Horizonte/MG, Brasil.

Maria Thereza Macedo Valadares - Rua Grão Pará, 926, aptº 404, Funcionários CEP 30150-341. Belo Horizonte/MG, Brasil. 
A expansão tecnológica ocorrida nas últimas décadas propiciou o desenvolvimento de todas as áreas de cuidados da saúde, modificando o perfil dos pacientes e das doenças. Na pediatria, o avanço tecnológico trouxe inegáveis progressos em todas as especialidades. Na neonatologia, os prematuros e recém-nascidos com baixo peso apresentam taxas de sobrevida cada vez maiores. Na infectologia, doenças antes consideradas prevalentes e graves são hoje pouco comuns, graças à vacinação e a medicamentos mais modernos. Na oncologia, o surgimento de novas terapêuticas permitiu significativa redução na mortalidade das crianças com câncer ${ }^{1-3}$.

Entretanto, apesar do aparato tecnológico, algumas crianças ainda vivem em condições que ameaçam a vida: as portadoras de sequelas graves ou que necessitam de cuidados especiais ou as que, em alguns casos, não respondem aos modernos tratamentos instituídos para suas doenças ${ }^{1,3}$. Lidar com esse novo perfil de paciente exige do pediatra uma abordagem diferente. Mesmo quando há tratamento curativo, cuidados paliativos devem e necessitam ser implementados, com o objetivo central de proporcionar melhor controle dos sintomas e melhor qualidade de vida para a criança e sua família.

Em 1998, a Organização Mundial da Saúde (OMS) apresentou uma definição específica para cuidados paliativos na pediatria: cuidado ativo e total prestado à criança, no contexto do seu corpo, mente e espírito, bem como o suporte oferecido a toda a sua família ${ }^{4}$. Segundo a OMS, o cuidado paliativo deve ser iniciado quando a doença crônica é diagnosticada, devendo caminhar concomitantemente com o tratamento curativo.

A avaliação e o alívio do sofrimento são prioridades nessa abordagem e devem ultrapassar o campo biológico, alcançando as esferas psíquica e social. Para que isso seja realmente possível, a OMS afirma a necessidade de equipe multidisciplinar que inclua, nesse processo, toda a família e o meio em que a criança está inserida. Tais cuidados podem ser prestados em centros de atenção primária, terciária e até mesmo no domicílio da criança ${ }^{4}$.

Hilmelstein destaca alguns princípios básicos para o cuidado paliativo infantil: o cuidado é focado na criança, orientado a toda família e construído com uma boa relação equipe-família. Deve-se avaliar individualmente cada criança, e respectiva família, respeitando suas crenças e valores e facilitando a comunicação. Tal cuidado deve estender-se após a morte, durante o luto familiar. Toda criança que tenha o diagnóstico de uma doença crônica, que ameaça à vida, deve receber cuidados paliativos.
Para o autor, essas condições podem se enquadrar em quatro grandes categorias: 1) condições nas quais o tratamento curativo é possível, mas pode haver falha terapêutica, como pacientes com doenças oncológicas; 2 ) condições que exigem tratamentos longos, com o objetivo de melhorar a qualidade de vida dos pacientes, como na fibrose cística; 3) doenças sabidamente progressivas, com tratamentos apenas paliativos, como a trissomia do 13 e do 18 e; 4) condições graves, não reversíveis, mas que implicam em muita vulnerabilidade, como as crianças com paralisia cerebral secundária decorrente de sofrimento fetal agudo ${ }^{5}$.

Para uma boa assistência a essas crianças, as intervenções não devem se limitar aos especialistas em cuidados paliativos. Pediatras gerais e de diversas áreas de atuação, como oncologistas, hematologistas ou neonatologistas, podem exercer de maneira efetiva esse cuidado, mediante conhecimento das necessidades da criança e de sua família e reconhecimento da importância de um trabalho multi e interprofissional, visando ao controle da dor e de outros sintomas, e a atenção individualizada e integral a cada paciente ${ }^{5,6}$.

\section{Método}

O trabalho baseia-se em revisão da literatura, com artigos indexados nas bases Lilacs e Medline via Pubmed. No Medline, foram utilizados os descritores: "Palliative Care" e "Hospice Care", com filtro para "all Child" e "Infant". A estratégia de busca foi: ("Palliative Care"[Majr]) OR "Hospice Care"[Majr]) AND ("infant"[Filter] OR "all child"[Filter]). Na base Lilacs a estratégia de busca foi "Cuidados Paliativos" OR "Hospice Care" OR "Cuidados a Doentes Terminais" OR "Cuidado Paliativo a Doentes Terminais" OR "Cuidados de Conforto" OR "Programas de Cuidados Intermitentes" OR "Programas de Cuidados Paliativos" OR "Assistência Paliativa" OR "Atención Paliativa" OR "Palliative Care" OR "Tratamento Paliativo", também com limite para a faixa etária pediátrica. A pesquisa foi sendo realizada à medida que a discussão sobre o tema foi se intensificando e novos estudos surgiam nos periódicos especializados. O levantamento foi efetivado de forma sistemática, incluindo estudos publicados entre janeiro de 2000 e outubro de 2012.

Foram incluídos apenas os artigos referentes à pediatria, em língua portuguesa e/ou inglesa, e excluídos os artigos que se limitavam à neonatologia e aqueles cujo termo paliativo não se referia à as- 
sistência, mas sim a tratamentos paliativos - como cirurgias cardíacas paliativas. De acordo com esses critérios, foram identificados 576 artigos. Todos os resumos foram lidos e discutidos pelos autores, que selecionavam os artigos mais relevantes. Além disso, foi realizada a verificação das referências citadas nos artigos selecionados. Ao final, 29 artigos foram utilizados para a presente revisão. Adicionalmente, utilizou-se o Manual de cuidados paliativos da Associação Nacional de Cuidados Paliativos, o atual Código de Ética Médica (2010) e o site eletrônico da Organização Mundial da Saúde.

\section{A prevalência e a abordagem dos sintomas}

O controle impecável da dor e de outros sintomas é a principal estratégia na abordagem das crianças que necessitam de cuidados paliativos. Mas são poucas as pesquisas sobre o manejo da dor e dos sintomas nos cuidados paliativos em pediatria, principalmente quando comparadas ao extenso leque de estudos referentes aos pacientes adultos ${ }^{5}$. Consequentemente, muitas das atuais recomendações são extrapoladas do universo adulto para o infantil.

Soma-se a essa lacuna o fato de que são muitas as crianças com necessidades de cuidados paliativos, mas a maioria dos estudos limita-se ao manejo dos sintomas prevalentes na criança com câncer ${ }^{5}$. Wolfe e colaboradores avaliaram os sintomas das crianças que morreram com câncer entre 1990 e 1997, no Dana Farber Institute e no Children's Hospital of Boston. Foram entrevistados os pais de 102 crianças que haviam falecido. Destas, quase $80 \%$ morreram da progressão da doença, enquanto as demais faleceram com complicações relacionadas ao tratamento.

Nesse estudo, de acordo com os pais, $89 \%$ das crianças apresentaram sofrimento significativo no último mês de vida, sofrimento esse secundário a pelo menos um sintoma, sendo os mais prevalentes a dor, a fadiga e a dispneia. Entre as crianças que apresentaram tratamento específico para os sintomas, o tratamento foi efetivo em apenas $27 \%$ das com dor e em $16 \%$ das com dispneia. Os pais relataram mais sintomas do que os registrados nos prontuários médicos ${ }^{7}$.

Em 2008, Wolfe comparou a prevalência dos sintomas das crianças do estudo acima com 119 crianças que evoluíram para o óbito, também secundário a neoplasia, entre 1997 e 2004 . A proporção de crianças com sintomas como fadiga, dor, dispneia e ansiedade não diferiu entre as duas coortes. Con- tudo, de acordo com os pais, houve menos relatos de sofrimento significativo secundário a dor, dispneia e ansiedade, o que sugeriu melhor abordagem dos sintomas pela equipe médica ${ }^{8}$.

Estudo similar foi realizado na Austrália, no Royal Children's Hospital de Melbourne, com questionários que também avaliaram o relato dos pais frente aos sintomas das crianças que faleceram com câncer, entre 1996 e 2004 . Entre as 96 famílias entrevistadas, $84 \%$ relataram sofrimento intenso vivenciado pela criança no último mês de vida, relacionado pelo menos a um sintoma: dor (46\%), fadiga (43\%) e hiporexia (30\%) foram os mais prevalentes. Dentre as crianças que receberam tratamento, apenas $47 \%$ obtiveram controle adequado da dor, $18 \%$ da fadiga e $17 \%$ da hiporexia ${ }^{9}$.

$\mathrm{Na}$ Holanda, Theunissen e colaboradores realizaram, entre 1999 e 2002, um estudo retrospectivo com pais de 32 crianças que faleceram com câncer em fase terminal, com vistas a verificar os sintomas físicos e também os psicológicos desses pacientes ${ }^{10}$. Os achados são equivalentes ao estudo de Wolfe ${ }^{7}$ e Jonh A. Heath ${ }^{9}$. De acordo com os pais, $82 \%$ dos sintomas físicos foram abordados pela equipe médica. Porém, apenas $18 \%$ e $26 \%$ desses sintomas apresentaram melhora completa ou parcialmente completa, respectivamente, após o manejo médico. Os sintomas psicológicos foram menos abordados pela equipe médica (43\%) e, após abordagem, apenas $9 \%$ e $25 \%$ tiveram melhora completa ou parcial, respectivamente ${ }^{10}$.

Por esses estudos fica evidente a necessidade de abordagem mais eficaz para o controle dos sintomas prevalentes na criança, objetivando alcançar o manejo ótimo da assistência paliativa. Limitações metodológicas, secundárias ao desenho retrospectivo dos estudos, devem ser consideradas. Além disso, nos estudos supracitados a percepção dos sintomas e de seu manejo adequado limitou-se à observação dos pais, o que pode não corresponder à percepção do médico ou da criança ${ }^{7,9}$.

Em 2004, Brian S. Carter realizou um estudo descritivo sobre a assistência médica prestada a crianças com doenças terminais. Foram incluídas 105 crianças que estavam hospitalizadas no momento da morte. Os dados foram obtidos a partir de relatos dos prontuários médicos referentes às últimas 72 horas de vida da criança. Entre os sintomas descritos com maior frequência estavam a dor (34\%) e a dispneia (24\%). Os autores destacaram, ainda, a diferença entre o registro médico dos sintomas e sua respectiva abordagem. Em relação à dor, aproximadamente $90 \%$ tiveram analgesia prescrita. Em relação à dispneia, apenas metade dos pacien- 
tes com esse sintoma recebeu tratamento específico. Nos pacientes com choro prolongado e fadiga, apenas a minoria recebeu tratamento ${ }^{11}$.

Ross Drake também avaliou a prevalência dos sintomas por meio do registro de prontuários médicos. Nesse estudo, foram incluídas 30 crianças que evoluíram para o óbito no Chidren's Hospital at Westmead, na Austrália. O número médio de sintomas por paciente na última semana de vida foi $11 \pm 5,6$, e seis sintomas apresentaram prevalência acima de 50\%: fadiga, sonolência, alterações dermatológicas, irritabilidade, dor e edema de membros inferiores ${ }^{12}$.

Collins e colaboradores, por sua vez, utilizaram uma escala de avaliação (Memorial Symptom Assessment Scale - MSAS) para determinar a prevalência dos sintomas em 159 crianças com câncer, com idade entre 10 e 18 anos, a partir do relato dos pacientes. Os sintomas mais prevalentes (acima de $35 \%$ ) foram fadiga, dor, sonolência, náusea, tosse, diminuição do apetite e sintomas psicológicos (tristeza, nervosismo, preocupação, irritabilidade). Os autores não encontraram diferença significativa na prevalência dos sintomas por idade ou sexo. A dor foi o sintoma mais prevalente no grupo dos pacientes hospitalizados (84\%), considerada muito angustiante por $52 \%$ das crianças ${ }^{13}$.

Independentemente do delineamento do estudo, observa-se a alta prevalência de sintomas nas crianças com necessidade de cuidados paliativos, especialmente próximo à data do óbito, quando esses cuidados são imprescindíveis. O relato dos pais é valiosa ferramenta para a abordagem dos sintomas, mas sempre que possível o relato da criança deve ser obtido, sendo considerado o "padrão-ouro" para a determinação dos sintomas ${ }^{6}$.

Em 2010, Jefrey C. Klick e Julie Hauer descreveram condições importantes para o manejo dos sintomas prevalentes no cuidado paliativo pediátrico. De acordo com os autores, uma parceria entre a equipe médica e os pais/criança é essencial para esclarecer os benefícios e os efeitos colaterais das medicações, com foco na melhor qualidade de vida possível para o paciente. Para o controle dos sintomas, tratamentos não farmacológicos (como musicoterapia, acupuntura, brincadeiras, massagens) podem ser úteis, especialmente se aliados às medidas farmacológicas ${ }^{6}$. Os autores destacam a relevância da abordagem holística dos sintomas (emocional, espiritual e social), haja vista que pode incrementar o sucesso do tratamento ${ }^{6}$.

Antecipar os sintomas, agir oportunamente e rever com frequência o resultado da abordagem dos mesmos são também princípios importantes ${ }^{6}$. Sabe-se que o manejo ineficaz dos sintomas apresentados pelas crianças está diretamente relacionado ao sofrimento substancial nos últimos dias de vida, vivenciado pela família e pelo paciente ${ }^{7,9}$. Otimizar essa assistência é o ponto-chave do cuidado paliativo.

\section{Equipe multiprofissional e comunicação com a família}

Atualmente, os cuidados paliativos estão intimamente ligados aos cuidados curativos, com foco na abordagem integral da criança e da família. Esse cuidado global requer a participação de equipe multidisciplinar, com médicos, enfermeiros, assistentes sociais, fisioterapeutas, terapeutas ocupacionais, religiosos, dentre outros ${ }^{14}$. Crianças com patologias variadas podem ser beneficiadas com cuidados paliativos e, consequentemente, profissionais de diversas áreas irão prestar esse tipo de assistência para seus pacientes ${ }^{15,1}$.

Atesta esta afirmação revisão retrospectiva realizada no Canadá, que avaliou sete programas de cuidados paliativos pediátricos e um hospice infantil. A maioria dos pacientes incluídos nesses programas tinha como diagnóstico primário alterações neurológicas $(39,1 \%)$, seguido por tumores malignos $(22,1 \%){ }^{15}$. Estudo em seis hospitais pediátricos americanos e canadenses, com 515 crianças, também mostrou que a condição clínica predominante era má-formação genética/congênita $(40,8 \%)$, seguida de doenças neuromusculares $(39,2 \%)$, câncer $(19,8 \%)$, doenças respiratórias $(12,8 \%)$ e gastrointestinais $(10,7 \%)^{16}$. Nos dois exemplos, a maioria dos pacientes era dependente de tecnologia médica, sendo o uso da gastrostomia $(48,5 \%)$ a forma mais comum ${ }^{17}$.

Na literatura, são poucos os estudos que avaliam a participação de uma equipe multi e interdisciplinar nos cuidados paliativos em pediatria. Em um estudo retrospectivo, Brian Carter avaliou as circunstâncias da morte de 105 crianças hospitalizadas, especialmente em relação ao controle dos sintomas e da participação de uma equipe pediátrica multidisciplinar. Durante a última internação desses pacientes, o acompanhamento multidisciplinar não foi documentado com frequência ${ }^{11}$.

Em 2009, Monterosso realizou entrevista sobre cuidados paliativos com 69 pais de crianças que faleceram com câncer. Concluiu que o cuidado dessas crianças e de suas famílias deve ser coordenado 
por equipe multidisciplinar e que se faz necessária a integração entre o serviço de atenção primária e terciária ${ }^{18}$. Diversos especialistas em cuidados paliativos pediátricos recomendam a participação de profissionais de diversas áreas na assistência às crianças com doenças crônicas. Devido a seu conhecimento específico, cada integrante da equipe fará abordagem específica, contribuindo para reduzir o sofrimento da criança e de seus familiares. Mas para que essa abordagem seja efetiva é necessária a comunicação clara entre os membros da equipe e a criança/família ${ }^{6}$.

Helle e Solomon entrevistaram os pais de 36 crianças com doenças que ameaçam a vida, que faleceram em três hospitais pediátricos universitários dos Estados Unidos. Para esses pais, a presença de equipe consistente durante toda a hospitalização favoreceu o bom relacionamento entre a família e os profissionais ${ }^{19}$. Estudo realizado por Jennifer W. Mack e colaboradores avaliou, sob a perspectiva dos pais e dos médicos, a qualidade do cuidado oferecido a crianças com câncer na fase final da vida. De acordo com os pais, a comunicação entre os médicos e a família foi considerada fator mais relevante para determinar a qualidade do cuidado médico ${ }^{20}$.

Em outro estudo, a qualidade da comunicação da equipe médica foi avaliada na perspectiva dos pais e também das crianças. Foram incluídas 20 crianças e adolescentes, com média de idade de 14,25 anos, com possibilidade de sobrevida menor que $20 \%$ em três anos. Os pais e os pacientes identificaram que a comunicação entre médico e família/ criança é o ponto-chave para a construção de um bom relacionamento ${ }^{21}$.

Meyer e colaboradores identificaram com 56 pais de crianças falecidas as prioridades para o cuidado pediátrico na fase final da vida com limitação de terapêutica invasiva e encontraram respostas similares: comunicação completa e verdadeira, fácil acesso à equipe, sentimento vivenciado e demonstrado pela equipe, preservação da integridade da relação pais-crianças e fé ${ }^{22}$.

Nobert J. Weidner afirma que na última década houve uma mudança cultural na prática médica: a abordagem paternalista foi substituída por outro modo de lidar com a relação, que reconhece o papel e a importância da família e do paciente. Para o autor, a medicina paliativa pediátrica deve reconhecer paciente e família com unidade central do cuidado ${ }^{23}$. Consistente com este novo critério, o Comitê de Bioética e Cuidados Hospitalares da Academia Americana de Pediatria (AAP) considera que os pediatras devem oferecer suporte aos pais e aos irmãos das crianças com doenças crônicas, pois todos são afetados pelo processo da doença.

Este comitê ressalta, também, que tanto os médicos quanto os pais devem conversar com a criança doente sobre seus sentimentos, medos e angústias. 0 desenvolvimento da criança, suas experiências anteriores com a morte, a religião e os aspectos culturais da família são fatores que devem ser considerados para tornar efetiva essa comunicação. Evitar esse tipo de comunicação é ignorar o fato de que as crianças doentes, na maioria das vezes, estão conscientes de sua situação. Para a Academia Americana de Pediatria, limitar terapêuticas fúteis não significa desejar ou antecipar o óbito das crianças, mas sim promover uma morte digna, sem dor e sem angústia ${ }^{15}$. Feudner salienta que o desenvolvimento de habilidades de comunicação beneficiará tanto os pacientes e familiares quanto a própria equipe médica ${ }^{24}$.

\section{Cuidados paliativos ao final da vida e limitação de terapêutica invasiva}

A Academia Americana de Pediatria defende o modelo integrado de cuidado paliativo: os componentes do cuidado paliativo são oferecidos no diagnóstico e continuam durante todo o curso da doença, quer a etapa final seja a cura ou a morte ${ }^{17}$. 0 acompanhamento da família durante o processo de morte e o luto, assim como o suporte para toda a equipe, também são ações dos cuidados paliativos ${ }^{1,15}$

Com a progressão da doença e a consequente redução das possibilidades curativas, os cuidados paliativos assumem uma curva ascendente, tornando-se necessidade absoluta ${ }^{25}$. Logo, assumir que não há lugar para cuidados paliativos até que as medidas curativas sejam exauridas pode interferir na abordagem precoce de questões delicadas, como limitação de terapêutica invasiva no fim da vida ${ }^{15}$.

Jennifer Mack afirma que abordagens médicas invasivas ainda fazem parte do cuidado de crianças com doenças progressivas, sem possibilidade de cura, inclusive próximo ao óbito, e que a integração precoce de cuidados paliativos no curso da doença facilita a preparação da família para a morte. Segundo a autora, entre as possíveis causas que justificam a persistência de medidas invasivas no final da vida está a dificuldade dos médicos em prever a morte, ou seja, reconhecer, de acordo com a trajetória da doença, se a morte está ou não próxima. Outra explicação plausível seria a comunicação inadequada com a família/paciente ${ }^{26}$. 
Em estudo realizado com 107 médicos, 71\% afirmaram que as discussões sobre questões relativas ao planejamento do cuidado de crianças com doenças ameaçadoras à vida ocorriam tardiamente. Os médicos também identificaram as principais barreiras que dificultavam essas discussões: expectativa irreal dos pais; diferença sobre o entendimento do prognóstico entre os pais/paciente e a equipe; e falta de preparo dos pais para discutir tais questões ${ }^{27}$.

No Brasil, ainda existe receio por parte de alguns médicos em relação à possibilidade de limitar terapêuticas invasivas para os pacientes com doenças progressivas. Entretanto, o atual Código de Ética Médica (CEM) ${ }^{28}$ explicita em vários artigos o dever do médico de oferecer cuidados paliativos aos pacientes com doenças incuráveis e terminais ${ }^{28}$ :

Artigo 41, parágrafo único - Nos casos de doença incurável e terminal, deve o médico oferecer todos os cuidados paliativos disponíveis sem empreender ações diagnósticas ou terapêuticas inúteis ou obstinadas, levando sempre em consideração a vontade expressa do paciente ou, na sua impossibilidade, a de seu representante legal ${ }^{29}$.

Tonelli e colaboradores avaliaram o perfil de assistência aos pacientes pediátricos que evoluíram para o óbito, em um hospital universitário. Os autores analisaram 106 casos e, desses, 51,9\% tiveram limitação de suporte de vida, sendo mais comum a limitação terapêutica na unidade neonatal e em pacientes com doenças limitantes da sobrevida ${ }^{30}$. Estudo realizado em três unidades de terapia intensiva pediátrica no Sul do Brasil também mostrou que $36,1 \%$ dos pacientes avaliados tiveram limitação do suporte de vida, observando-se associação entre limitação de suporte de vida e doença crônica ${ }^{31}$.

Em 2007, o mesmo grupo fez um estudo sobre os cuidados ao final da vida em crianças no Brasil e no mundo. Os autores afirmam que existem diferenças em relação à limitação de suporte de vida em vários países, que se relacionam a fatores religiosos, culturais, legais e econômicos. Observaram que no Brasil, nos últimos anos, houve aumento da limitação de suporte de vida nas unidades de tratamento intensivo pediátrico, de $6 \%$ para $40 \%$, sendo a ordem de não reanimar a forma mais frequente ${ }^{32}$.

É importante ressaltar, porém, que nesses estudos de Tonelli e Lago a participação da família nas decisões sobre limitação de terapêutica correspondeu a $20,8 \%$ e menos de $10 \%$, respectivamente ${ }^{29,30}$. No entanto, Himelstein ${ }^{5}$ ressalta que a qualidade do cuidado em pediatria está relacionada ao compartilhamen- to das decisões médicas entre a família, a criança e a equipe, o que permitiria esperar percentuais significativamente mais elevados. $\mathrm{O}$ autor destaca a necessidade de envolver os adolescentes enfermos nesse processo de decisões médicas, sempre que possível. A Academia Americana de Pediatria também apoia a participação dos pacientes nas discussões ${ }^{15,33}$.

Jefferson Piva ${ }^{1}$ publicou em 2011 artigo de revisão sobre os dilemas e as dificuldades envolvendo decisões de final de vida e cuidados paliativos em pediatria. $\mathrm{O}$ autor esclarece que, assim como proposto por Himelstein ${ }^{5}$, as decisões sobre limitação de terapêutica fútil devem ser compartilhadas com o paciente e a família (como no caso de crianças e pacientes incapazes).

$O$ autor sugere que antes de envolver a participação da família no processo decisório é necessária a existência de consenso entre a equipe responsável pelo paciente. A partir desse momento, Piva recomenda que sejam identificadas e excluídas as intervenções fúteis (ações que não irão contribuir para o controle da doença e não irão resultar em melhor qualidade de vida para o paciente) e determinadas prioridades terapêuticas individualizadas, como otimização da analgesia. $\mathrm{O}$ autor relata que as condutas frente a possíveis intercorrências/complicações do quadro devem ser previamente discutidas com a família e registradas de maneira clara nos prontuários médicos ${ }^{1}$.

\section{Considerações finais}

A literatura mostra que, atualmente, a terapia paliativa não se encontra mais no polo oposto da terapêutica curativa. A integração dos dois modelos pode proporcionar melhor qualidade de vida para as famílias e para as crianças com doenças crônicas e ameaçadoras à vida. Reconhecer e tratar efetivamente os sintomas mais prevalentes nos pacientes, participar de equipe multidisciplinar, desenvolver bom relacionamento entre a equipe, a família e os pacientes e discutir as questões relacionadas ao final da vida são alguns fundamentos básicos dos cuidados paliativos.

Seja na atenção primária ou terciária, todo profissional de saúde irá lidar com esse novo perfil de paciente. Assim, instituir essa temática durante todo o processo de formação do médico e, especificamente, dos pediatras é uma necessidade real e absoluta, que irá beneficiar a toda a equipe envolvi$\mathrm{da}$, mas, principalmente, os pacientes, contribuindo para vida e morte dignas. 


\section{Referências}

1. Piva JP, Garcia PCR, Lago PM. Dilemas e dificuldades envolvendo decisões de final de vida e oferta de cuidados paliativos em pediatria. [Internet]. Rev. bras. ter. intensiva. 2011 (acesso 18 jul. 2013);23(1):78-86. Disponível: http://www.scielo.br/scielo.php?script=sci_arttext\&pid=S0103507X2011000100013\&lng=en\&nrm=iso

2. Moritz RD, Deicas A, Capalbo M, Forte DN, Kretzer LP, Lago P et al. II Fórum do grupo de estudos do fim da vida do Cone Sul: definições, recomendações e ações integradas para cuidados paliativos na unidade de terapia intensiva de adultos e pediátrica. [Internet]. Rev. bras. ter. intensiva. 2011 (acesso 16 jul. 2013);23(1):24-9. Disponível: http://www.scielo.br/scielo.php?pid=S0103507X2011000100005\&script=sci_arttext

3. Floriani CA. Cuidados paliativos no domicílio: desafios aos cuidados de crianças dependentes de tecnologia. [Internet]. J. pediatr. 2010 (acesso 18 jul. 2013);86(1):15-20. Disponível: http://www. scielo.br/pdf/jped/v86n1/v86n1a04.pdf

4. World Health Organization. Cancer pain relief and palliative care in children. [Internet]. Geneva: WHO; 1998 (acesso 18 jul. 2013). Disponível: http://whqlibdoc.who.int/publications/9241545127.pdf

5. Himelstein BP. Palliative care for infants, children, adolescents, and their families. J Palliat Med. 2006;9(1):163-81.

6. Klick JC, Hauer J. Pediatric palliative care. [Internet]. Curr Probl Pediatr Adolesc Health Care. 2010 (acesso 16 jul. 2013);40(6):120-51. Disponível: http://www.ncbi.nlm.nih.gov/pubmed/20638035

7. Wolfe J, Grier HE, Klar N, Levin SB, Ellenbogen JM, Salem-Schatz S et al. Symptoms and suffering at the end of life in children with cancer. [Internet]. N Engl J Med. 2000 (acesso 18 jul. 2013);342(5):326-33. Disponível: http://www.ncbi.nlm.nih.gov/pubmed/10655532

8. Wolfe J, Hammel JF, Edwards KE, Duncan J, Comeau M, Breyer J et al. Easing of suffering in children with cancer at the end of life: is care changing? [Internet]. J Clin Oncol. 2008 (acesso 15 jul. 2013);26(10):1717-23. Disponível: http://www.ncbi.nlm.nih.gov/pubmed/?term=Easing+of+ suffering+in+children+with+cancer+at+the+end+of+life\%3A+is+care+changing\%3F

9. Heath JA, Clarke NE, Donath SM, McCarthy M, Anderson VA, Wolfe J. Symptoms and suffering at the end of life in children with cancer: an Australian perspective. [Internet]. Med J Aust. 2010 (acesso 18 jul. 2013);192(2):71-5. Disponível: http://www.ncbi.nlm.nih.gov/pubmed/?term=Symptoms+an $\mathrm{d}+$ suffering+at+the+end+of+life+in+children+with+cancer\%3A+an+Australian+perspective

10. Theunissen JM, Hoogerbrugge PM, Van Achterberg T, Prins JB, Vernooij-Dassen MJ, Van den Ende $\mathrm{CH}$. Symptoms in the palliative phase of children with cancer. [Internet]. Pediatr Blood Cancer. 2007 (acesso 16 jul. 2013);49(2):160-5. Disponível: http://www.ncbi.nlm.nih.gov/ pubmed/16972239

11. Carter BS, Howenstein M, Gilmer MJ, Throop P, France D, Whitlock JA. Circumstances surrounding the deaths of hospitalized children: opportunities for pediatric palliative care. [Internet]. Pediatrics. 2004 (acesso 17 jul. 2013);114(3):361-6. Disponível: http://pediatrics.aappublications. org/content/114/3/e361.full.pdf+html

12. Drake R, Frost J, Collins JJ. The symptoms of dying children. [Internet]. J Pain Symptom Manage. 2003 (acesso 18 jul. 2013);26(1):594-603. Disponível: http://www.ncbi.nlm.nih.gov/ pubmed/12850642

13. Collins JJ, Byrnes ME, Dunkel IJ, Lapin J, Nadel T, Thaler HT et al. The measurement of symptons in children with cancer. [Internet]. J Pain Symptom Manage. 2000 (acesso 18 jul. 2013);19(5):36377. Disponível: http://www.ncbi.nlm.nih.gov/pubmed/10869877

14. Michelson KN, Steinhorn DM. Pediatric end-of-life issues and palliative care. [Internet]. Clin Pediatr Emerg Med. 2007 (acesso 18 jul. 2013);8(3):212-9. Disponível: http://www.ncbi.nlm.nih. gov/pubmed/18438449

15. American Academy of Pediatrics. Committee on Bioethics and Committee on Hospital Care: palliative care for children. [Internet]. Pediatrics. 2000 (acesso 16 ago. 2013);106:351-7. Disponível: http://www.ncbi.nlm.nih.gov/pubmed/10920167

16. Widger K, Davies D, Drouin DJ, Beaune L, Dauost L, Farran P et al. Pediatric patients receiving palliative care in Canada. Arch Pediatr Adolesc Med. 2007;161(6):597-602.

17. Feudtner $\mathrm{C}$, Kang $\mathrm{TI}$, Hexem KR, Friedrichsdorf SJ, Osenga K, Siden $\mathrm{H}$ et al. Pediatric palliative care patients: a prospective multicenter cohort study. [Internet]. Pediatrics. 2011 (acesso 16 jul. 2013);127(6):1.094-101. Disponível: http://www.ncbi.nlm.nih.gov/pubmed/21555495

18. Monterosso L, Kristjanson LJ, Phillips MB. The supportive and palliative care needs of Australian families of children who die from cancer. [Internet]. Palliat Med. 2009 (acesso 18 jul. 2013);23(6):526-36. Disponível: http://www.ncbi.nlm.nih.gov/pubmed/19351793

19. Heller KS, Solomon MZ. Continuity of care and caring: what matters to parents of children with life-threatening conditions. [Internet]. J Pediatr Nurs. 2005 (acesso 18 jul. 2013);20(5):335-46. Disponível: http://www.ncbi.nlm.nih.gov/pubmed/16182093

20. Mack JW, Hilden JM, Watterson J, Moore C, Turner B, Grier HE et al. Parent and physician perspectives on quality of care at the end of life in children with cancer. [Internet]. J Clin Oncol. 2005 (acesso 18 jul. 2013);23(36):9.155-61. Disponível: http://www.ncbi.nlm.nih.gov/ pubmed/16172457 
21. Hsiao JL, Evan EE, Zeltzer LK. Parent and child perspectives on physician communication in pediatric palliative care. [Internet]. Palliat Support Care. 2007 (acesso 18 jul. 2013);5(4):355-65. Disponível: http://www.ncbi.nlm.nih.gov/pubmed/18044413

22. Meyer EC, Ritholz MD, Burns JP, Truog RD. Improving the quality of end-of-life care in the pediatric intensive care unit: parents' priorities and recommendations. [Internet]. Pediatrics. 2006 (acesso 16 jul. 2013);117(3):649-57. Disponível: http://www.ncbi.nlm.nih.gov/pubmed/16510643

23. Weidner NJ. Pediatric Palliative Care. [Internet]. Curr Oncol Rep. 2007 (acesso 18 jul. 2013); 9(6):437-9. Disponível: http://www.ncbi.nlm.nih.gov/pubmed/17991350

24. Feudtner C. Collaborative communication in pediatric palliative care: a foundation for problem-solving and decision-making. [Internet]. Pediatr Clin North Am. 2007 (acesso 18 jul. 2013);54(5):583-607. Disponível: http://www.ncbi.nlm.nih.gov/pubmed/17933613

25. Pinto AC, Cunha AA, Othero MB, Bettebga RT, Barbosa SM, Chiba T et al. Manual de cuidados paliativos. [Internet]. Rio de Janeiro: Diagraphic; 2009. Disponível: http://www.nhu.ufms.br/ Bioetica/Textos/Morte\%20e\%20o\%20Morrer/MANUAL\%20DE\%20CUIDADOS\%20PALIATIVOS. pdf

26. Mack JW, Wolfe J. Early integration of pediatric palliative care: for some children, palliative care starts at diagnosis. [Internet]. Curr Opin Pediatr. 2006 (acesso 18 jul. 2013);18(1):10-4. Disponível: http://www.ncbi.nlm.nih.gov/pubmed/16470155

27. Durall A, Zurakowski D, Wolfe J. Barriers to conducting advance care discussions for children with life-threatening conditions. [Internet]. Pediatrics. 2012 (acesso 16 jul. 2013);129(4):975-82. Disponível: http://pediatrics.aappublications.org/content/129/4/e975

28. Conselho Federal de Medicina. Resolução CFM n 1.965, de 17 setembro de 2009. Código de ética médica. [Internet]. (acesso 18 jul. 2013). Disponível: http://portal.cfm.org.br/index. php?option=com_content\&view=category\&id=9\&Itemid $=122$

29. Conselho Federal de Medicina. Op. cit. p.51.

30. Tonelli HAF, Mota JAC, Oliveira JS. Perfil das condutas médicas que antecedem ao óbito de crianças em um hospital terciário. [Internet]. J pediatr. 2005 (acesso 18 jul. 2013);81(2):118-25. Disponível: http://www.scielo.br/pdf/jped/v81n2/v81n02a06.pdf

31. Lago PM, Piva J, Kipper D, Garcia PC, Pretto C, Giongo M et al. Limitação de suporte de vida em três unidades de terapia intensiva pediátrica do sul do Brasil. [Internet]. J Pediatr. 2005;81(2):1117. Disponível: http://www.scielo.br/pdf/jped/v81n2/v81n02a05.pdf

32. Lago PM, Devictor D, Piva JP, Bergounioux J. Cuidados de final de vida em crianças: perspectivas no Brasil e no mundo. [Internet]. J pediatr. 2007 (acesso 17 jul. 2013);83(2 Suppl):S109-16. Disponível: http://www.scielo.br/pdf/jped/v83n2s0/a13v83n2s0.pdf

33. Hinds PS, Drew D, Oakes LL, Fouladi M, Spunt SL, et al. End-of-life care preferences of pediatric patients with cancer. J Clin Oncol. 2005 (acesso 18 jul. 2013);23(36):9.146-54. Disponível: http:// www.ncbi.nlm.nih.gov/pubmed/16172453

\section{Participação dos autores}

Todos tiveram colaboração na revisão bibliográfica e no processo de redação e edição do artigo.

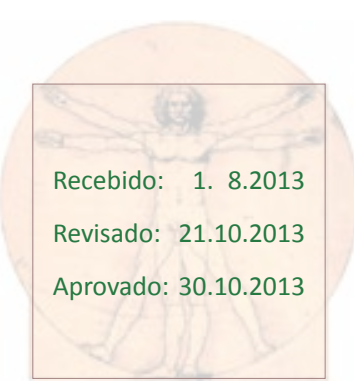

